\title{
Successful oxytocin-assisted nipple aspiration in women at increased risk for breast cancer
}

\author{
Karijn P. M. Suijkerbuijk • Elsken van der Wall • \\ Helen Meijrink - Xiaojuan Pan - Inne H. M. Borel Rinkes • \\ Margreet G. E. M. Ausems · Paul J. van Diest
}

Published online: 5 May 2010

(C) The Author(s) 2010. This article is published with open access at Springerlink.com

\begin{abstract}
The high rate of interval malignancies urges for new screening methods for women at high risk for breast cancer. Nipple aspiration provides direct access to the breast tissue and its DNA, and therefore is a likely candidate, but clinical applications have been limited by the failure to obtain nipple aspiration fluid from most women. We performed oxytocin-assisted nipple aspiration in 90 women at increased risk for breast cancer based on family history or genetic test results $(n=63)$ and/or previous breast cancer $(n=34)$. Nipple fluid was obtained from $81 / 90$ women $(90 \%)$ and bilaterally in $77 \%$. Mean discomfort rating was 0.6 (on a $0-10$ scale), which was significantly lower than for mammography or MRI. These findings suggest that a new tool for biomarker detection in oxytocin-assisted nipple fluid of women at high risk for breast cancer is at hand.
\end{abstract}

Keywords Nipple aspiration - Breast cancer - BRCA1 . BRCA2 $\cdot$ Screening $\cdot$ Early detection

K. P. M. Suijkerbuijk $\cdot$ H. Meijrink $\cdot$ X. Pan

P. J. van Diest $(\bowtie)$

Department of Pathology, University Medical Center Utrecht, PO Box 85500, 3508 GA Utrecht, The Netherlands

e-mail: P.J.vanDiest@umcutrecht.nl

E. van der Wall

Division of Internal Medicine and Dermatology, University

Medical Center Utrecht, 3508 GA Utrecht, The Netherlands

I. H. M. Borel Rinkes

Department of Surgical Oncology, University Medical Center Utrecht, 3508 GA Utrecht, The Netherlands

M. G. E. M. Ausems

Department of Medical Genetics, University Medical Center Utrecht, 3508 GA Utrecht, The Netherlands

\begin{abstract}
Abbreviations
DCIS Ductal carcinoma in situ

NAF Nipple aspirate fluid

QM-MSP Quantitative multiplex methylation specific PCR

RPFNA Random periareolar fine needle aspiration
\end{abstract}

\section{Introduction}

Breast cancer is the most prevalent cancer for women in the Western world, accounting for 519,000 deaths worldwide each year [1]. Approximately one in eight women will eventually develop breast cancer, but for some women this risk is even higher. A hereditary predisposition is the most important risk factor, with a lifetime risk of developing breast cancer up to $80 \%$ in women known to carry a BRCA1 or BRCA2 germline mutation [2].

Several screening and prevention options are available to these women, in order to either prevent the development of breast cancer or detect it at an early stage. However, until now, screening programs are only partly effective and in one out of four in high-risk women interval malignancies are detected [3]. Prophylactic mastectomy and salpingooophorectomy have been proven to effectively decrease the risk in women at high risk, but the morbidity associated with this procedure discourages many women to choose this option.

A screening method that gives access to the tissue in which breast cancer arises could add value to the existing methods and increase the rate of breast cancer detection at an early stage [4]. Nipple Aspirate Fluid (NAF), containing breast epithelial cells, free DNA, secreted proteins and hormones is produced in the breast ducts of non-lactating 
women. In the last decades, multiple techniques have been developed to obtain this fluid, such as non-invasive nipple aspiration, ductal lavage and Random Periareolar Fine Needle Aspiration (RPFNA). Nipple aspiration was previously reported to be only feasible in a small part of the population $[5,6]$. The other two techniques showed higher success percentages, but their invasive nature is a serious drawback.

From a previous pilot study we reported that, using oxytocin nasal spray, we were able to obtain nipple fluid in $94 \%$ out of 67 healthy volunteers, of which $75 \%$ yielded fluid bilaterally [7]. The procedure was very well tolerated. Here we report the initial results of a prospective study on oxytocin-assisted nipple aspiration in women with a moderate to high breast cancer risk.

\section{Materials and methods}

\section{Study population}

This study is part of a project that aims to explore the possibilities of monitoring development of epigenetic changes in nipple aspirates, with the ultimate goal to optimally schedule preventive (or refrain from) breast surgery in high-risk women. To this end we are assessing promoter hypermethylation in nipple aspirates of high-risk women, of which some will undergo preventive or therapeutic surgery. This will enable assessing the correlation between methylation patterns and the development of (pre)malignant lesions in this group. Oxytocin-assisted nipple aspiration is performed annually. Besides, participants will undergo regular radiological and/or clinical examination as they were used to. Timing of breast surgery will be agreed between patient and oncologist based on usual clinical and radiological findings, blinded to the results of the nipple aspirate analyses. The study protocol of this study was approved by the Hospital's Ethics Committee and written informed consent was obtained from all participants. This report is a summary of the baseline nipple aspiration experience of the first 90 subjects enrolled between August 2008 and July 2009.

\section{Eligibility criteria}

Women were considered at increased risk and included in this study if they were carriers of a $B R C A 1$ or $B R C A 2$ germline mutation, had a pedigree-based risk $\geq 20 \%$, or had a history of Ductal Carcinoma in Situ (DCIS) or invasive breast cancer. Pregnancy and bilateral mastectomy were exclusion criteria. We did not exclude women that previously underwent other forms of breast surgery, radiotherapy, chemotherapy or hormonal treatment.
Nipple aspiration procedure

Anesthetic cream (Emla) was applied onto the nipple while the breasts were warmed for $10 \mathrm{~min}$. The subjects were administered 4 IE of oxytocin into both nostrils. The nipple was gently wiped with a dekeratinizing agent in order to remove keratin plugs and subsequently cleansed with ethanol. A suction cup (aspirator) was placed over the nipple. Repeated gentle suction by a syringe $(10-50 \mathrm{cc})$ drew fluid to the nipple surface, where droplets were collected by capillary tubes. The entire procedure was repeated at the other breast. Quantitative Multiplex Methylation Specific PCR (QM-MSP) for RASSF1A, SCGB3Al and APC was performed on 50 randomly selected nipple fluid samples obtained in this study as described before [8].

\section{Questionnaires}

A questionnaire, addressing breast cancer risk factors and factors that might influence the success of the procedure was filled out before nipple fluid aspiration. After aspiration participants rated the experienced discomfort during different stages of the procedure and other screening procedures from 0 ("no discomfort at all") to 10 ("the worst I can imagine").

\section{Statistical analysis}

A paired $T$-test was used to compare the discomfort scores of different procedures. Fisher's exact and Chi-square tests were used to examine the association between NAF yielding and potential predictive values. For this analysis, subgroups from Table 1 were pooled because of low numbers. A selection of independent variables (see Table 3) was used in a logistic regression analysis using backward selection procedure, retaining covariates with $P \leq 0.10$, for the right and the left breast separately. SPSS 15.0 for Windows was used for statistical analysis, regarding two-sided $P \leq 0.05$ as significant.

\section{Results}

A total of 90 women at increased risk were included in this study. Sixty-three women had a family history of breast cancer, six of them were known carriers of a germline BRCA1 mutation and six had a BRCA2 mutation. Twelve women had a history of DCIS and 22 women previously had invasive breast cancer. Participants were 21-72 years old (mean 48 years). All baseline characteristics are shown in Table 1.

The procedure was very well endured. Mean discomfort rating for the total procedure was 0.6, which was 
Table 1 Patient characteristics of 90 patients at increased risk for invasive breast cancer undergoing oxytocin-assisted nipple fluid aspiration

$n(\%)$

Age (years)

$<40$

$22(24 \%)$

$40-49$

$\geq 50$

$25(28 \%)$

$43(48 \%)$

BRCA germline mutation

None detected

BRCA1

BRCA2

BRCA1 in family ${ }^{\mathrm{a}}$

BRCA2 in family ${ }^{\mathrm{a}}$

Pedigree-based risk

None

Moderate (20-30\%)

High (30-40\%)

Known mutation (> 40\%)

Personal history of breast cancer

No

\section{DCIS}

Invasive carcinoma

Spontaneous nipple discharge

Yes

No

Number of live births

0

$1-2$

$\geq 3$

Age at first birth (years)

$<25$

25-29

$\geq 30$

Age at menarche (years)

$$
\begin{aligned}
& <12 \\
& 12-14
\end{aligned}
$$

$>14$

Menopausal status

Premenopausal

Postmenopausal

Menstrual cycle day ${ }^{\mathrm{b}}$

$$
\text { 1-7 }
$$

7-14

14-21

$>21$

Age at menopause (years)

$$
<45
$$

$45-50$

$>50$

$28(31 \%)$

$6(7 \%)$

$6(7 \%)$

$2(2 \%)$

$1(1 \%)$

$27(30 \%)$

$12(13 \%)$

$17(19 \%)$

$18(20 \%)$

$56(62 \%)$

$12(13 \%)$

$22(24 \%)$
$27(30 \%)$

$29(32 \%)$

$32(36 \%)$

$42(47 \%)$

$48(53 \%)$

$12(13 \%)$

$4(4 \%)$

$9(10 \%)$

$11(12 \%)$

$16(18 \%)$

$16(18 \%)$

$16(18 \%)$

Table 1 continued

$n(\%)$

Previous breast feeding

Yes

$51(57 \%)$

No

$39(43 \%)$

Current oral contraceptive use

Yes

$14(16 \%)$

No

$76(84 \%)$

Current hormonal replacement therapy

Yes

$2(2 \%)$

No

$88(98 \%)$

Size breast

Small (A-B)

$24(27 \%)$

Normal (C-D)

$58(64 \%)$

Large $>$ D

$8(9 \%)$

Prophylactic oophorectomy

Yes

$18(20 \%)$

No

$72(80 \%)$

Previous chemotherapy

Yes

$12(13 \%)$

No

$78(87 \%)$

Previous radiotherapy

Yes

$23(26 \%)$

No

$67(74 \%)$

Breast surgery

None

$50(56 \%)$

Excision biopsy

$6(7 \%)$

Breast conserving

$16(18 \%)$

Ablation

$17(19 \%)$

Other

$1(1 \%)$

a These women have a 50\% risk of being a carrier, but have chosen not to undergo testing (yet)

b Six premenopausal women did not have a menstrual cycle, due to contraceptives (hormonal Intrauterine Device etc.)

Table 2 Discomfort of nipple aspiration compared to other screening procedures for 90 patients at high risk for invasive breast cancer undergoing oxytocin-assisted nipple fluid aspiration

\begin{tabular}{llll}
\hline & $\begin{array}{l}\text { Number } \\
\text { of women }\end{array}$ & Mean score (SE) & $P$-value \\
\hline Nipple aspiration & 90 & $0.6(0.1)$ & \multicolumn{1}{c}{} \\
Breast feeding & 51 & $1.8(0.3)$ & $<0.001$ \\
Physical examination & 83 & $1.1(0.2)$ & 0.004 \\
Mammography & 83 & $4.9(0.3)$ & $<0.001$ \\
MRI & 31 & $2.6(0.4)$ & $<0.001$ \\
\hline
\end{tabular}

${ }^{a}$ Paired samples $T$-test 
significantly lower than breast feeding (1.8), physical examination by a medical oncologist or surgeon (1.1), MRI (2.6) and mammography (4.9; Table 2). No side effects were reported.

Nipple fluid was obtained from 81 out of 90 women (90\%) and bilaterally in $77 \%$. QM-MSP for RASSF1, $S C G B 3 A 1$ and $A P C$ was possible in all 50 samples that we have analyzed. From the 15 women that were known to carry a $B R C A 1$ or $B R C A 2$ mutation or that have family members that are known to be carriers, 14 women yielded bilaterally upon aspiration; in one woman nipple aspiration was only successful in one breast. Characteristics that made women significantly less likely to yield nipple fluid upon aspiration, were not having a family history of breast cancer $(P=0.002)$, postmenopausal status $(P=0.03)$ and previous surgery (excision biopsy, breast conserving surgery or other surgery of the breast) on the right side of the breast $(P=0.002$; Table 3$)$. Age did not significantly influence the success of the procedure $(P=0.08)$.

Using logistic regression, no factor could be identified that significantly contributed to the success of nipple aspiration in the left breast in multivariate analysis. For the right breast, only an increased pedigree-related risk (B 2.6; $P=0.02)$ correlated to the success rate of the aspiration in a multivariate model.

\section{Discussion}

Here we describe our findings of performing oxytocinassisted nipple aspiration in a cohort of 90 high-risk women. We were able to obtain nipple fluid in $90 \%$ of high-risk women not selected for age, hormonal factors or previous therapy. A negative effect of surgery of the right breast on NAF yielding was shown, but this effect was not significant in the left breast. Furthermore, in 56\% of women that previously underwent surgery of the right breast aspiration was still successful. Therefore, previous surgery is not a reason to exclude women from our study. Similarly, although postmenopausal status and not having a family history for breast cancer decreased the likelihood to yield NAF, $83 \%$ of postmenopausal women and $78 \%$ of women without a family history of breast cancer still yielded NAF.

A recent article by Loud et al. [9] reported failure to obtain nipple fluid (not supported by oxytocin) in the large majority of women from BRCA families. In this study, only 45 out of 171 women from BRCA families (26\%) yielded NAF, 63\% (107/171) could have a duct cannulated for ductal lavage, while cytologically evaluable samples were obtained in only $41 \%$ of these women. Women without a prior breast cancer history had an increased likelihood of yielding NAF and the presence of NAF was
Table 3 Predictive factors for successful oxytocin-assisted nipple aspiration in 90 patients at increased risk for invasive breast cancer undergoing nipple fluid aspiration

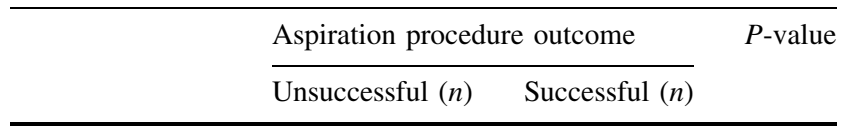

Pedigree-based risk

Not increased 61

Increased $\quad 0 \quad 47$

$\mathbf{0 . 0 0 2}^{\mathrm{a}}$

Spontaneous nipple discharge

Yes $\quad 0 \quad 11$

$\begin{array}{lll}\text { No } & 9 & 70\end{array}$

Parity

Nulliparous $\quad 2 \quad 28$

$\begin{array}{lll}\text { Parous } & 7 & 53\end{array}$

Menopausal status

Premenopausal 111

$\begin{array}{llll}\text { Postmenopausal } & 8 & 40 & \mathbf{0 . 0 3}^{\mathrm{a}}\end{array}$

Previous breast feeding

$\begin{array}{lll}\text { Yes } & 7 & 44\end{array}$

$\begin{array}{llll}\text { No } & 2 & 37 & 0.29^{\mathrm{a}}\end{array}$

Current oral contraceptive

$\begin{array}{lll}\text { Yes } & 0 & 14\end{array}$

$\begin{array}{llll}\text { No } & 9 & 67 & 0.35^{\mathrm{a}}\end{array}$

Previous chemotherapy

$\begin{array}{lll}\text { Yes } & 2 & 10\end{array}$

$\begin{array}{llll}\text { No } & 7 & 71 & 0.34^{\mathrm{a}}\end{array}$

Previous radiotherapy

$\begin{array}{lll}\text { Yes } & 3 & 20\end{array}$

$\begin{array}{llll}\text { No } & 6 & 61 & 0.69^{\mathrm{a}}\end{array}$

Breast surgery left ${ }^{\mathrm{b}}$

$\begin{array}{lll}\text { Yes } & 6 & 16\end{array}$

$\begin{array}{llll}\text { No } & 8 & 51 & 0.15^{\mathrm{c}}\end{array}$

Breast surgery right ${ }^{\mathrm{b}}$

$\begin{array}{llrl}\text { Yes } & 7 & 9 & \\ \text { No } & 7 & 59 & \mathbf{0 . 0 0 2}^{\text {c }}\end{array}$

a Fisher's exact test

b Ablation cases were excluded for this analysis (analysis for left and right breast separately)

c Chi-square test

an independent correlate of ductal lavage cell count adequacy. From these data, the authors concluded that ductal lavage is unlikely to be useful in breast cancer screening among $B R C A 1 / 2$ mutation carriers. 
The present study provides quite different results. We believe that vacuum-assisted nipple aspiration, if feasible, is to be preferred over ductal lavage because of its noninvasive nature. Unlike most previous studies, we do not assess cytology, but are focusing on methylation aberrations in our nipple fluid samples. As reported by Loud et al. [9], multiple studies have questioned the reproducibility and clinical value of cytology in nipple fluid. Moreover, Fackler et al. [10] showed that, compared to cytology, analyzing promoter methylation in ductal lavage cells doubled the detection rate of breast cancer. In a previous study, Dooley et al. [11] were able to obtain nipple fluid in a comparable cohort in $84 \%$ of women, but in this study only $27 \%$ yielded adequate samples for diagnosis.

In conclusion, from our experience oxytocin-assisted nipple aspiration is a very well tolerated non-invasive procedure to obtain nipple fluid in virtually all women, including BRCA1 and BRCA2 mutation carriers. The high success rate of the procedure is not restricted by age, reproductive factors or previous therapy and suggests that a new tool for biomarker detection in nipple fluid of women at increased risk for breast cancer is at hand.

Acknowledgments Financial support: This work was supported by the Dutch Cancer Society (UU 2007-3977 and UU 2008-4217), the "Integraal Kankercentrum Midden-Nederland (IKMN)", the "Ata Visser Stichting", the American Women's Club of The Hague and A Sister's Hope/Pink Ribbon.

Conflict of interest statement We declare no conflict of interest for any of the authors.

Open Access This article is distributed under the terms of the Creative Commons Attribution Noncommercial License which permits any noncommercial use, distribution, and reproduction in any medium, provided the original author(s) and source are credited.

\section{References}

1. World Health Organization (2009). http://www.who.int/media centre/factsheets/fs297/en/index.html Cited 6 Jan 2010

2. King MC, Marks JH, Mandell JB (2003) Breast and ovarian cancer risks due to inherited mutations in BRCA1 and BRCA2. Science 302(5645):643-646

3. Brekelmans CT, Seynaeve C, Bartels CC et al (2001) Effectiveness of breast cancer surveillance in BRCA1/2 gene mutation carriers and women with high familial risk. J Clin Oncol 19(4): 924-930

4. Suijkerbuijk KP, van der Wall E, Vooijs M, van Diest PJ (2008) Molecular analysis of nipple fluid for breast cancer screening. Pathobiology 75(2):149-152

5. Fabian CJ, Kimler BF, Mayo MS, Khan SA (2005) Breast-tissue sampling for risk assessment and prevention. Endocr Relat Cancer 12(2):185-213

6. Wrensch MR, Petrakis NL, Gruenke LD et al (1990) Factors associated with obtaining nipple aspirate fluid: analysis of 1428 women and literature review. Breast Cancer Res Treat 15(1): $39-51$

7. Suijkerbuijk KP, van der Wall E, van Diest PJ (2007) Oxytocin: bringing magic into nipple aspiration. Ann Oncol 18(10): 1743-1744

8. Fackler MJ, McVeigh M, Mehrotra J et al (2004) Quantitative multiplex methylation-specific PCR assay for the detection of promoter hypermethylation in multiple genes in breast cancer. Cancer Res 64(13):4442-4452

9. Loud JT, Thiebaut ACM, Abati AD et al (2009) Ductal lavage in women from BRCA1/2 families: is there a future for ductal lavage in women at increased genetic risk of breast cancer? Cancer Epidemiol Biomarkers Prev 18(4):1243-1251

10. Fackler MJ, Malone K, Zhang Z et al (2006) Quantitative multiplex methylation-specific PCR analysis doubles detection of tumor cells in breast ductal fluid. Clin Cancer Res 12(11): 3306-3310

11. Dooley WC, Ljung BM, Veronesi U et al (2001) Ductal lavage for detection of cellular atypia in women at high risk for breast cancer. J Natl Cancer Inst 93(21):1624-1632 